\title{
Adopting Multiview Pixel Mapping for Enhancing Quality of Holoscopic 3D Scene in Parallax Barriers Based Holoscopic 3D Displays
}

\author{
M.R Swash, O. Abdulfatah, E. Alazawi, T. Kalganova and J. Cosmas \\ School of Engineering and Design, Brunel University London, UK
}

\begin{abstract}
The Autostereoscopic multiview 3D Display is robustly developed and widely available in commercial markets. Excellent improvements are made using pixel mapping techniques and achieved an acceptable 3D resolution with balanced pixel aspect ratio in lens array technology. This paper proposes adopting multiview pixel mapping for enhancing quality constructed holoscopic 3D scene in parallax barriers based holoscopic 3D displays achieving great results. The Holoscopic imaging technology mimics the imaging system of insects, such as the fly, utilizing a single camera, equipped with a large number of micro-lenses, to capture a scene, offering rich parallax information and enhanced 3D feeling without the need of wearing specific eyewear. In addition pixel mapping and holoscopic 3D rendering tools are developed including a custom built holoscopic 3D displays to test the proposed method and carry out a like-to-like comparison.
\end{abstract}

Index Terms - Holoscopic, Integral image, Rendering, Autostereoscopic, 3D, lens array, Lenticular, Parallax barrier, 3D Display, Pixel mapping, Unidirectional, viewpoint, Display, 3DTV

\section{INTRODUCTION}

$\mathrm{T}$ HREE dimensional display technology is a topical research topic and a well-sought product in the commercial display market today. The 3D resolution including constructed $3 \mathrm{D}$ image quality remains a challenge in $3 \mathrm{D}$ display technology, namely Autostereoscopic Multiview [1], Holographic [2][3] and Holoscopic - also known as integral imaging [4][5]. In multiview 3D display, the pixel mapping techniques are used to achieve 2D like pixel aspect ratio as well as advancing horizontal 3D resolution. This is achieved by trailing vertical resolution and gaining in horizontal resolution. Unidirectional autostereoscopic 3D displays require more pixels in the horizontal direction and the horizontal resolution is divided by number of pixels per lens (viewpoint images), which provides the horizontal 3D resolution. As a result the $3 \mathrm{D}$ pixel aspect ratio gets unbalance and the pixel mapping techniques [6][7] are applied to achieve an almost square or wide screen pixel aspect ratio and also improve horizontal 3D resolution in multiview 3D displays. In this paper, the multiview pixel mapping technique [7] is adapted to improve the holoscopic 3D image quality in parallax barriers based holoscopic 3D display.

The parallax barriers based holoscopic 3D display uses pinhole array sheet to project viewpoint pixels into the space where a virtual $3 \mathrm{D}$ scene is constructed by intersection points of viewpoint pixels. It is identified that parallax barriers technology has many advantages over lens array i.e. Parallax barriers offer switchable 2D/3D as well as content adaptive 3D displays e.g. possibility is there that pinholes can be resized in runtime therefore pixels per pinhole can be configured in the user preference [17][18][19]. In addition, in parallax barriers technology, viewpoint pixels are propagated correctly into the space with minimum noise, whereas lens array introduce noises and has limited view angles, which depend on the lens array manufacturing material

In Holoscopic imaging, a 3D scene is constructed by collective pinhole/lens arrays which project viewpoint-pixels into the space where the intersection point of two viewpointpixels creates a 3D pixel as shown in Fig 1. Viewpoint-pixels are projected successively, therefore, increasing the number of pixels per pinhole growths the distance between two pinholes "dark space", which reduces 3D resolutions and also introduces blackness to the $3 \mathrm{D}$ scene. As a result, Parallax barriers based 3D displays can be come very dim compared to the lens array based 3D displays and this is the main reason, lens arrays are used widely to build 3D displays.

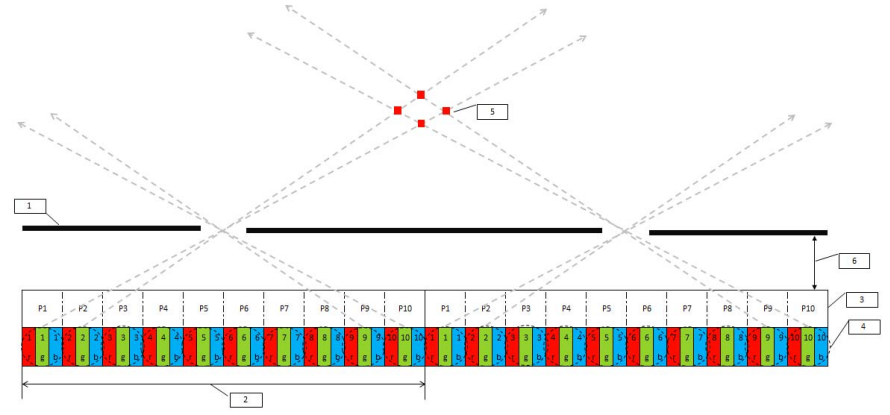

Fig 1. Viewpoint pixels representation (1: Pinhole array, 2: Pinhole pitch size, 3: Pixels per pinhole, 4: Viewpoint RGB dot pixel structure, 5: 3D pixel 'viewpoint pixels Intersections', 6: Focal length)

The proposed method opens a new avenue for designing parallax barriers based holoscopic 3D displays with natural enough 3D image quality and lighting. It is an ideal candidate for improving the dimness of parallax barriers $3 \mathrm{D}$ display and $3 \mathrm{D}$ resolution improvement is a bonus too. 


\section{Adopting Multiview PiXel MapPing FOR Holoscopic}

The autostereoscopic multiview 3D display [1] is developed based on Stereo 3D displays [8]. The main difference is that it projects more than two perspective views in slightly different angles using lens array or parallax barrier technologies, in so that users' eyes see the convenient views. Multiview 3D displays technology has a sweet spot as well as minimum and maximum distance where users perceives $3 \mathrm{D}$ effects. To date, all multiview 3D displays are unidirectional for instance a 8view multiview $3 \mathrm{D}$ display will have a $3 \mathrm{D}$ resolution of $2 \mathrm{D}$ width resolution $/ 8 \times 2 \mathrm{D}$ height resolution, which creates a very unbalanced pixel aspect ratio problem and also accommodating more views/viewers will increase the unbalance pixel aspect ratio further. This problem is overcome by applying pixel mapping techniques [6][7].

The pixel mapping technique separates sub-pixels (Red, Green, Blue sub-pixel) of a view pixel in the vertical direction, using the vertical resolutions to enhance the horizontal resolution as shown in Fig 2. This is because a sub-pixel size is used to project a single pixel data. Thus more 3D pixels can be fitted compared to the traditional approach and also the lens array gets slanted as the sub-pixels are distributed in the vertical direction.

In particular, the pixel mapping techniques have been applied for multiview 3D displays and our recent paper [9] discusses about parsing a holoscopic 3D image format to multiview 3D displays compatible format, in which case, the Philips pixel mapping is implemented [6]. In this paper, the focus is on adopting the multiview pixel mapping technique such as Alioscopy's pixel mapping [7] for improving constructed holoscopic 3D image quality as well as holoscopic 3D resolution in Parallax barriers based holoscopic 3D displays. The Alioscopy pixel mapping [7] method spreads a viewpoint pixel into 3 rows by shifting horizontally as illustrated in Fig 2. This process creates $18.43^{\circ}$ degrees slanting, therefore the lens array needs to be slanted by $18.43^{\circ}$ degrees. As it fits a pixel into a subpixel, it triples the horizontal $3 \mathrm{D}$ resolution where the vertical resolution is reduced to $1 / 3$ and the $3 \mathrm{D}$-DPI remains unchanged. As adopting multiview pixel mapping in Parallax barriers based holoscopic 3D display, inherits the advantages for example, it makes the 3D display size to growth in both horizontal and vertical directions, when more $3 \mathrm{D}$ pixels are added. Also the horizontal 3D resolution is enhanced compared to the traditional method. More importantly, it enhances the quality of the constructed holoscopic 3D image.

The pixel mapping method reduces vertical 3D resolutions and enhances horizontal 3D resolutions. For instance, Fig 2 illustrates the pixel mapping, which is implemented in Alioscopy displays [7] that divides the vertical 3D resolutions by 3 and multiplies the horizontal 3D resolutions by 3; because it uses a physical subpixel size to project a pixel by shifting RGB subpixel in vertically.

This paper discusses adopting multiview pixel mapping for enhancing the dimness of parallax barriers based holoscopic 3D display. As shown in Fig 3, the parallax barriers overlaid on the pixel sheet, which shows how a pinhole is mapped to its elemental image. The process and structure is exactly the same for parallax barriers. Having said that, pinhole mapping is different because in this case the pinhole array is not rotated, unlike lens arrays; instead subpixel size rectangular pinholes are created and shifted away to create the slanting angle.

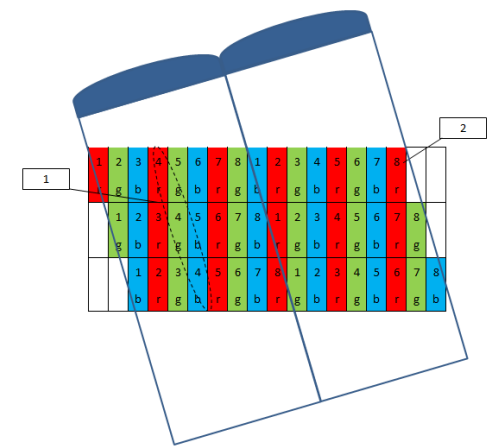

Fig 2. State of the art pixel mapping techniques [6][7] (1: construction of a single viewpoint pixel, a: number of pixels per lens)

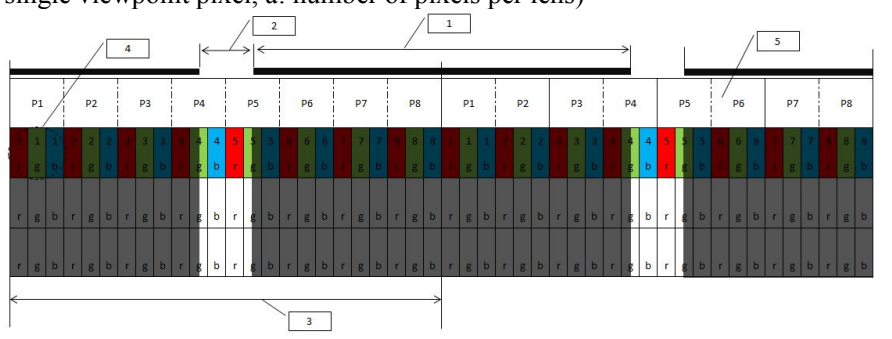

(a)

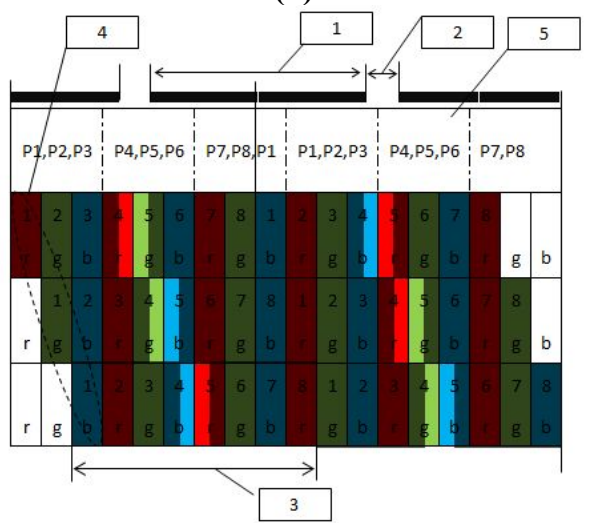

(b)

Fig 3. Pixel mapping structure in parallax barriers - (a) classical parallax barriers based holoscopic 3D Display, (b) proposed pixel mapped parallax based holoscopic 3D display

It can be argued that the dark areas between two pinholes are reduced to $1 / 3$ and also the pinhole pitch is reduced by $1 / 3$ and then the overall lighting versus black areas remains the same; which is true but in the tradition approach, there are 3 times less 3D pixels in horizontal direction shown in Fig (1) compared to the proposed method shown in Fig (2). Therefore dark areas are dominant as $3 \mathrm{D}$ pixels are very shallow in the space. The proposed approach triples 3D pixels in the space and in other words, it triples 3D pixels per inch (DPI), which improves the overall 3D image lighting quality.

\section{HOLOSCOPIC PIXEL PREPARATION}

Holoscopic pixels/subpixels are remapped according to the supported pixel mapping method and slanted by $18.43^{\circ}$ degrees to achieve a compatible 3D image. The main difference is that $3 \mathrm{D}$ resolutions of holoscopic $3 \mathrm{D}$ images are 
different because the proposed method has more horizontal resolution and less vertical resolution than the traditional method.

In the preparation stage, holoscopic pixels are distributed vertically by shifting the subpixels in horizontally. Fig 3.(b) illustrates the distribution of the Holoscopic pixels in both vertical and horizontal directions under each pinhole to create new compatible pixels. For instance the first pixel: $\mathrm{R}$ subpixel is placed in row 1, column 1; G sub-pixel is placed in row 2, column 2; and B sub-pixel is placed in row 3, column 3 according to Equation 1. This subpixel distribution process also creates a $18.43^{\circ}$ degrees slanting shape which matches to the lens array slanting shown in Fig 3.

Eq 1. A Newly created pixel contains subpixels of 3different viewpoint pixels

$$
\text { Pixel }^{\mathrm{r}, \mathrm{c}}<=\mathrm{R}^{\text {Pixel[i] }}+\mathrm{G}^{\text {Pixel[i+1] }}+\mathrm{B}^{\text {Pixel[i+3] }}
$$

Where $\mathrm{r}=$ row; $\mathrm{c}=$ column; $\mathrm{R}=$ red; $\mathrm{G}=$ green; $\mathrm{B}=$ blue;

The display has 5040 subpixels in the horizontal direction where each microlens has 10 pixels that are mapped to 10 physical subpixels and it has 504 pinholes in total. Equation 2 shows a 3D pixel resolution of $504 \times 3503 \mathrm{D}$ pixels with aspect pixel ratio of 1.44 which is almost wide screen aspect pixel ratio standard. These parameters are taken under consideration while rendering a Holoscopic 3D image.

Eq 2. The display 3D pixels counts

$(1680 * 3) /(10)=5043 \mathrm{D}$ pixels in horizontal direction

$1050 / 3=350$ pixels in vertical direction

\section{Holoscopic 3D IMAge ReNDERING}

A Holoscopic 3D camera model is developed that is based on Lippmann's approach [10], in which images are captured through a lens array. Detailed discussion on microlens images contributing to generating a single $3 \mathrm{D}$ image is reported in $[10][11][12][13][14][15] . \quad$ The concept of building a holoscopic 3D camera based on orthographic projections in computer graphics is patented by $\mathrm{BBC}$ [13]. In this setup, the holoscopic 3D camera is built using 10 multiple orthographic cameras which renders 10 orthographic viewpoint images in POV-Ray[16] and then the viewpoint images are interlaced to form a Holoscopic 3D image.

As shown in Table 1 and Table 2, by taking into account the parallax barriers parameters including the display, holoscopic 3D images are rendered and the rendering step are as follows:

1. Render 10 orthographic viewpoint images with resolution of $504 \times 350$ pixels

2. Apply Interlacing algorithm to interweave 10 viewpoint images to create a holoscopic 3D image with resolution of $5040 \times 350$ pixels

3. Apply the developed pixel mapping algorithm to remap the holoscopic 3D image pixels to the compatible pixel representation and create a 3D image with resolution $1680 \times 1050$ pixels

The prepared 3D images are opened in full screen mode, which can be done by any media player. This is done in order not to execute any type of 3D player and the display size with its parameters taken into consideration during the rendering and preparation. Therefore when the $3 \mathrm{D}$ image is opened in full screen mode, it fits well to the display resolution and the pinhole array alignment is carried out manually (human hand). As a result, a small noise is expected as the alignment accuracy is $+/-20$ microns. The alignment is very important to avoid crosstalk noise and other errors in 3D scene construction process.

\section{THE HOLOSCOPIC 3D DisPlay}

We have built the holoscopic 3D displays in the lab to test the prepared holoscopic 3D image and the compare the results of both the proposed and classical holoscopic 3D displays. The custom built holoscopic 3D displays enable us to carry out a like-2-like comparison. The holoscopic 3D displays have exactly the same hardware specifications including pixel pitch size, pixels per pinhole, and focal length as shown in Table 1 and Table 2 . The proposed holoscopic 3D display has 3 times more pinhole arrays than the traditional one because it fits 10 pixels (30 Subpixels) in the size of 10 physical subpixels size. Therefore the constructed holoscopic 3D image is sharper and brighter compared to the traditional one. The proposed method enhanced the holoscopic 3D scene quality by tripling the horizontal resolution.

Table 1. The traditional holoscopic 3D display specification

\begin{tabular}{|l|l|}
\hline \multicolumn{1}{|c|}{ Items } & \multicolumn{1}{c|}{ Specification } \\
\hline Active display area & $473.76(\mathrm{H}) \times 296.1(\mathrm{~V}) \mathrm{mm}$ \\
\hline Pixel pitch & $0.282(\mathrm{H}) \times 0.282(\mathrm{~V}) \mathrm{mm}$ \\
\hline 2D Resolution & $1680 \times 1050$ pixels \\
\hline Pixel arrangement & RGB vertical stripe \\
\hline Backlight source & $250 \mathrm{~cd} / \mathrm{m} 2$ \\
\hline Pixels per pinhole & $10 \mathrm{pixels}$ \\
\hline Pinhole pitch & $10 \times 0.282 \mathrm{~mm}=2.82 \mathrm{~mm}$ \\
\hline Pinhole sheet & 168 pinholes \\
\hline
\end{tabular}

Table 2. The proposed holoscopic 3D display specification

\begin{tabular}{|l|l|}
\hline \multicolumn{1}{|c|}{ Items } & \multicolumn{1}{c|}{ Specification } \\
\hline Active display area & $473.76(\mathrm{H}) \times 296.1(\mathrm{~V}) \mathrm{mm}$ \\
\hline RGB Pixel pitch & $0.282(\mathrm{H}) \times 0.282(\mathrm{~V}) \mathrm{mm}$ \\
\hline 2D Resolution & $1680 \times 1050$ pixels \\
\hline Pixel arrangement & RGB vertical stripe \\
\hline Backlight source & $250 \mathrm{~cd} / \mathrm{m} 2$ \\
\hline Pixels per pinhole & 10 pixels \\
\hline Pinhole pitch & $10 \times(0.282 \mathrm{~mm} / 3)=0.94 \mathrm{~mm}$ \\
\hline Pinhole sheet & 504 pinholes \\
\hline
\end{tabular}

\section{The Playback Result}

After the holoscopic 3D images were rendered and prepared, we replayed the 3D images on the custom built holoscopic 3D displays and as seen the resulting image playback in Fig 4, the result is very promising and the dark spacing barriers between two pinholes is not visible with a naked eye. Also it does improve the constructed holoscopic 3D image quality (see the visual richness quality of earth in both images). 


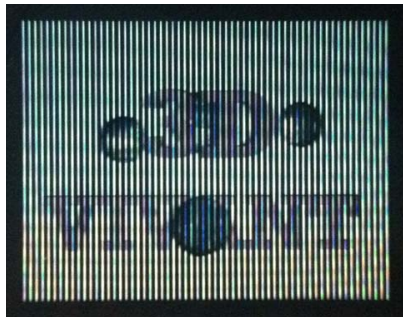

(a)

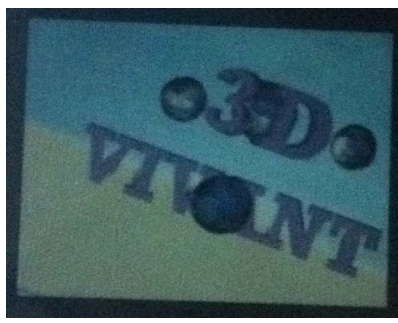

(b)
Fig 4. Playback result of proposed method (b) vs. tradition method (a)

\section{CONCLUSION}

In this paper, we have proposed and discussed the adoption of multiview pixel mapping in parallax barriers based holoscopic 3D display for enhancing quality of holoscopic 3D scene as well as improving the backlighting. The experiment is conducted on custom built parallax barriers based 3D displays. The experiment result was promising as dark areas between two pinholes are reduced (3 times smaller) and the overall holoscopic 3D image quality is improved significantly. We have built custom holoscopic 3D displays to perform like2like experiment and have also developed a virtual holoscopic 3D camera with a 3D pixel mapping module, which renders a computer graphics holoscopic 3D image and applies multiview pixel mapping technique. The prepared holoscopic 3D image is replayed directly by opening it in full screen mode. To sum up, the proposed method adopts multiview pixel mapping techniques and applies them on a holoscopic 3D image, which is then replayed newly built parallax barriers based holoscopic 3D display.

\section{ACKNOWLEDGMENT}

This work has been supported by European Commission under Grant FP7-ICT-2009-4 (3DVIVANT). The authors wish to ex-press their gratitude and thanks for the support given throughout the project.

\section{REFERENCES}

[1] Y. Zhang, Q. Ji, and W. Zhang, "Multi-view autostereoscopic 3D display," Optics Photonics and Energy Engineering (OPEE), vol. 1, pp. 58-61, 2010.

[2] P. S. Hilaire, S. A. Benton, and M. Lucente, "Synthetic aperture holography: a novel approach to threedimensional displays," J. Opt. Soc. Am. A, vol. 9, no. 11, pp. 1969-1977, Nov. 1992.

[3] M. Lucente, "Interactive three-dimensional holographic displays: seeing the future in depth," ACM SIGGRAPH Computer Graphics, no. May, 1997.

[4] Y. Kim, J.-H. Park, H. Choi, S. Jung, S.-W. Min, and B. Lee, "Viewing-angle-enhanced integral imaging system using a curved lens array.," Optics express, vol. 12, no. 3, pp. 421-9, Feb. 2004.

[5] M. C. Forman, A. Aggoun, and M. McCormick, "A novel coding scheme for full parallax 3D-TV pictures," 1997 IEEE International Conference on Acoustics, Speech, and Signal Processing, pp. 2945-2947, 1997.
[6] Van B. Hove and J. A. Clarke, "Autostereoscopic Display Appa-ratus,", U.S. Philips Corporation, New York, N.Y 2000.

[7] Alioscopy, "Alioscopy 3D Display Pixel Mapping Principles," Alioscopy, 2013. [Online]. Available: http://www.alioscopy.com/en/principles.php. [Accessed: 01-May-2013].

[8] H. Choi, "Current status of stereoscopic 3D LCD TV technolo-gies," 3D Research, vol. 2, no. 2, pp. 2-5, Nov. 2011.

[9] M.R. Swash, A. Aggoun, O. Abdulfatah, B. Li, J. C. Fernández, and E. Tsekleves, "Holoscopic 3D image rendering for Autostereoscopic Multiview 3D display," IEEE international Symposium on Broadband Multimedia Systems and Broadcasting, 2013.

[10] G. Lippmann, 'Epreuves reversibles', Comptes rendus hebdom-adaires des Seances de l'Academie des Sciences 146, 446-451, March 1908

[11] G. Milnthorpe, M. McCormick, A. Aggoun, and N. Davies, "Computer generated content for 3D TV displays," International Broadcasting Convention, 2002.

[12] M.R. Swash, A. Aggoun, O. Abdulfatah, B. Li, J. C. Fernández, and E. Tsekleves, "Omnidirectional Holsocopic 3D Content Generation Using Dual Orthographic Projection," IEEE international Symposium on Broadband Multimedia Systems and Broadcasting, 2013.

[13] G. A. Thomas and R. F. Stevens, "Processing of Images for 3D Display," U.S. Patent US6798409B22004

[14]J. Ren, A. Aggoun, and M. McCormick, "Computer generation of integral 3D images with maximum effective viewing zone," Proceedings of SPIE, vol. 5006, pp. 6573, 2003.

[15]M. Eljdid, A. Aggoun, and O. Youssef, "Computer Generated Content for 3D TV," 3DTV Conference, 2007, pp. 1-4, 2007.

[16] Persistence of Vision Pty. Ltd. (2004), Persistence of Vision Raytracer (Version 3.6). [Online]. Available: http://www.povray.org/download/

[17] Dekker, T., de Zwart, S. T., Willemsen, O. H., Hiddink, M. G. H., \& IJzerman, W. L. (2006). 2D/3D switchable displays, 61350K-61350K-11. doi:10.1117/12.657545

[18]Harrold, J., Wilkes, D., \& Woodgate, G. (n.d.). Switchable 2D/3D Display-Solid Phase Liquid Crystal Microlens Array. Proc. IDW, 2-3. Retrieved from http://www.ocuity.co.uk/Ocuity_IDW_04.pdf

[19]Liang, D., Luo, J.-Y., Zhao, W.-X., Li, D.-H., \& Wang, Q.-H. (2012). 2D/3D Switchable Autostereoscopic Display Based on Polymer-Stabilized Blue-Phase Liquid Crystal Lens. Display Technology, Journal of, 8(10), 609-612. doi:10.1109/JDT.2012.2209398

[20]McCormick, M., Davies, N. A., Milnthorpe, G., Aggoun, A., \& Forman, M. C. (2002). Integral imaging as a modality for 3D TV and displays. In B. Javidi \& F. Okano (Eds.), Society of Photo-Optical Instrumentation Engineers (SPIE) Conference Series (Vol. 4864, pp. 5159). 